\title{
The Influence of Surface Energy on the Washing Quality of Filter Cakes
}

\author{
Markus Wilkens, Urs A. Peuker \\ Technische Universität Bergakademie Freiberg Institute of Mechanical Process Engineering and \\ Mineral Processing, Freiberg, Germany \\ E-mail: markus.wilkens@mvtat.tu-freiberg.de
}

Received May 1, 2011; revised May 30, 2011; accepted June 9, 2011

\begin{abstract}
The washing of filter cakes, especially the displacement washing, represents an important aspect in science researches and industrial applications. A lot of ongoing researches are focussed on impurities, which are dissolved in the mother liquid (e.g. sodium chloride) and washed out with the identical pure liquid without impurities. The project flushing focuses on systems with two chemically different liquids. The main aim is to exchange an organic solvent by water. This article focuses on the adsorption effects during a washing process with solid systems of different wetting behaviours.
\end{abstract}

Keywords: Filter Cake Washing, Surface Energy Effects, Residual Content

\section{Introduction}

Washing processes and in this case also flushing processes always involve the elution of impurities. The aim is the preferably complete replacement of the mother liquid by the lowest possible amount of washing liquid.

A possibility to characterise a washing process is the washing curve (Figure 1), here a concentration ratio $c / c_{0}$ is plotted as a function of a washing ratio $W$. The concentration ratio is related to the concentration $c_{0}$ of the impurity at the beginning of the washing process (for a flushing process the concentration of the organic solvent in the mother liquid (normally 100\%)). The concentration $c$ represents the actual concentration of the impurity. This concentration $c$ can be related to the filtrate or the filter cake itself. The washing ratio $W$ represents the ratio of the added washing liquid volume related to the volume of the pores in a fully saturated filter cake. In case of a flushing process a mass washing ratio is used, because of the difficult calculation of the washing liquid volume for two-liquid-systems. Due to this, the mass of the added washing liquid $M_{W}$ is related to the mass of mother liquid in the filter cake pores $M_{\mathrm{V}}$ at the beginning of the flushing process.

$$
W_{M}=\frac{M_{W}}{M_{V}}
$$

Because of the fact, that the concentration of an impu- rity within the filter cake is not detectable, the concentration ratio in the filter becomes an inaccessible factor. For the industrial application a possibility has to be found, to calculate the concentration ratio in the filter cake out of an accessible factor (e.g. the concentration ratio in the filtrate). Due to this Heuser [1] defined the residual content $R$ as an integral factor for the concentration ratio in the filter cake.

$$
R=1-\int_{W=0}^{W} c^{*} \mathrm{~d} W
$$

The concentration factor $c^{*}$ represents the concentra-

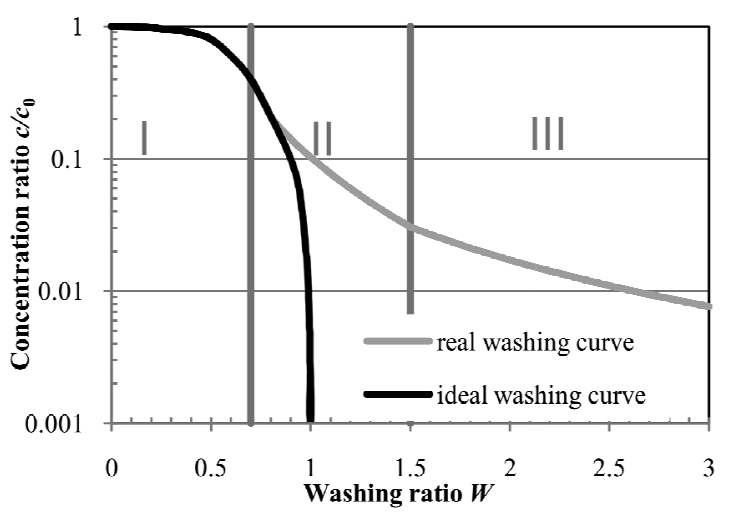

Figure 1. Ideal and exemplary real washing curve (concentration ratio in the filter cake depending on the washing ratio). 
tion ratio $c / c_{0}$ in the filtrate and a factor for the adsorption of the mother liquid in the filter cake.

In Figure 1 an ideal washing curve and an exemplary real washing curve are shown. For an ideal washing process or flushing process a complete exchange of the mother liquid by the washing liquid happens at a washing ratio of 1 . This means it is possible to clean the filter cake with the same amount of washing liquid as the amount of mother liquid in the filter cake.

The real washing curve is divided in three characteristic areas $[2,3]$; the displacement regime (I), the interstitial regime (II) and the diffusion regime (III). During the displacement regime the washing liquid displaces the mother liquid in a kind of a plug flow. Due to this only mother liquid is detected in the washing filtrate. The end of displacement regime is indicated by the detection of a concentration ratio lower than one in the washing filtrate. At this point a mixture of mother and washing liquid leaves the filter cake and the interstitial regime starts. During the last regime of the washing process (III) the removal of the mother liquid is dominated by diffusion mechanisms (diffusion regime). The remaining impurities in the filter cake diffuse out of stagnant areas into the primary stream. This removal often occurs slowly because of sterical barriers. Quite long time scales are needed to achieve a significant impurity removal [4].

\section{Materials}

For the research of the adsorption properties on a washing process an aluminosilicate (Dorkafill, $x_{50}=1.65 \mu \mathrm{m}$ ) in three different surface modifications is used. The surfaces vary from hydrophilic (DF600) over hydrophilichydrophobic (DF601) to hydrophobic (DF602). Because of the identical shape and particle size distribution the filtration and flushing are only influenced by structure effects, which result from the different particle-fluid interactions [5].

The two liquids water and methanol are used as mother and washing liquid. Characteristic of this system is that the progression of the dynamic viscosity depends on the mixing ratio of water and methanol, with a maximum at a methanol percentage of 40 vol.-percent.

\section{Methods}

\subsection{Filtration}

The filter cake formation is performed with a lab scale suction filter (nutsch filter) according to VDI 2762. The filtration area is $19.64 \mathrm{~cm}^{2}$ (diameter $50 \mathrm{~mm}$ ). The process parameters are a solid volume concentration of 0.2 and a filtration pressure of 3 bar. For the displacement washing a fully saturated filter cake is needed, because of this the filtration is stopped when the suspension reaches the filter cake. With the known porosity of each system it is possible to calculate the amount of liquid staying in the filter cake, which is necessary for the calculation of the washing ratio during the flushing step.

\subsection{Flushing}

For the flushing process the filter cake (inside a cake formation unit) is removed from the suction filter into a combined compression-washing unit.

Before and during the washing the filter cake is compressed by a pneumatic piston with a constant pressure of 3.5 bar to prevent fissuring and crack formation. The washing liquid flow is provided by a pressure difference of 2 bar.

For the composition analyses of the washing liquid (concentration ratio in the filtrate) samples in an interval of $\Delta W=0.05$ are taken. A Karl-Fischer-Titrator and a gas chromatograph (GC) are used for the analysis.

\section{Results}

For the technical control of a flushing process the concentration of the impurity (organic solvent) in the filtrate is in most cases the only possibility to get online informations of achieved washing qualities after a certain time or washing ratio.

Figure 2 shows the concentration ratio $c / c_{0}$ in the filtrate depending on the mass washing ratio for the hydrophilic DF600.

Due to the hydrophilic surface of DF600 the surface energy between water and DF600 is higher than between methanol and DF600. The definition of the surface energy as the ratio of the needed work to create a new surface and the new surface itself shows, that there is a

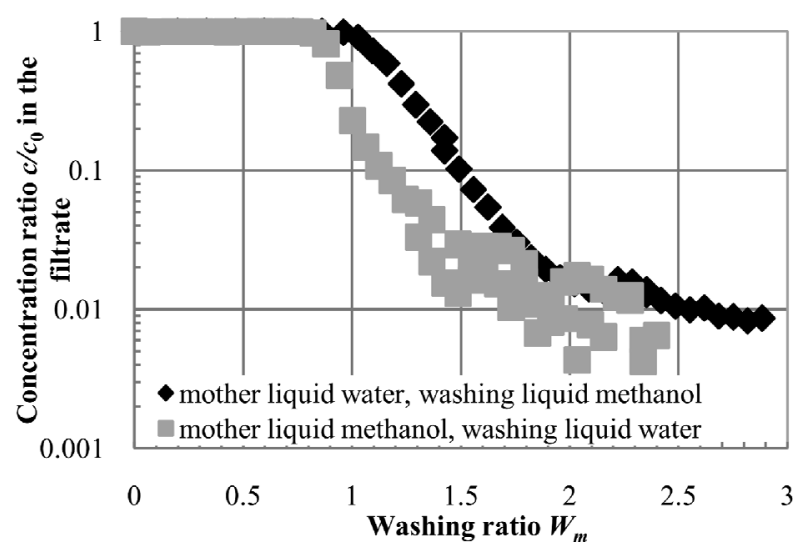

Figure 2. Flushing process of DF600 (hydrophilic) with water and methanol. 
preferential exchange of a liquid with a poor wetting behaviour by a liquid with a good wetting behaviour.

This fact correlates with the lower reachable concentration ratios of the washing process with methanol as mother liquid and water as washing liquid (grey plot).

Between the two washing directions (1 mother liquid water, 2 mother liquid methanol) a shift of the washing regimes can be observed. For methanol as mother liquid the displacement regime ends at a washing ratio of 0.81 , for water at a washing ratio of 0.96 . Because of the different structures of the filter cakes (porosities, pore size distribution) this shift can not be directly ascribed to the different surface energies. If no preferred adsorption of the two liquids exists, there should be no shift between the washing regimes and the washing quality, represented by the concentration ratios.

The system DF601 with a hydrophilic-hydrophobic surface shows such behaviour.

Both flushing steps show an identical washing curve, with the end of the displacement regime in a range of $W$ $=0.87$.

This fact leads to the assumption that the better the wettability of a system the better is the washing result, independent on the structure behaviours.

Until now only the concentration ratios in the washing filtrate are presented. The more interesting concentration in the filter cake can be calculated by the residual content. For the experimental work a possibility is found to extract the liquid of a flushed filter cake by a freezing unit, which is based on the principle of a vacuum cooling trap.

Figure 4 shows the residual content of the hydrophilic system DF600 with methanol as mother liquid and water as washing liquid.

A comparison between the residual content according to Heuser (black) and the residual content experimentally determined shows a difference of nearly one dimension for high washing ratios. This would lead to a wrong technical dimensioning of flushing processes. The residual content calculated in consideration of the influence of the volume flow fluctuation [6,7] during a flushing process leads to a relative good fit between calculation and experiment. This new kind of residual content is based on the residual content expressed by Heuser.

$$
R_{\text {new }}=R \cdot\left(1-\frac{\int_{0}^{W(t)} \frac{V_{\text {Filtrate }}\left(W_{m}(t)\right)}{W_{m}(t)} \mathrm{d} W_{m}(t)}{\int_{0}^{W_{\max }} \frac{V_{\text {Filtrate }}\left(W_{m, \max }\right)}{W_{m, \max }} \mathrm{d} W_{m, \text { max }}}\right)
$$

Thought has been given to an amplification of the residual content $\mathrm{R}$ by a normalised volume flow. In future works more attention will be paid to the volume flow fluctuation and the effects which cause the fluctuation.

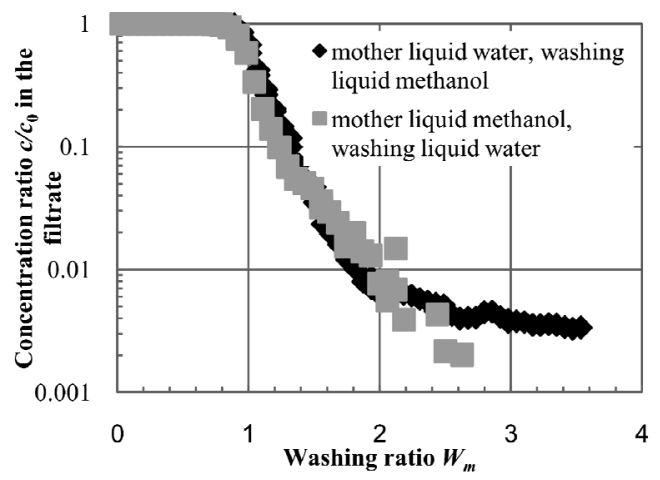

Figure 3. Flushing process of DF601 (hydrophilic-hydrophobic) with water and methanol.

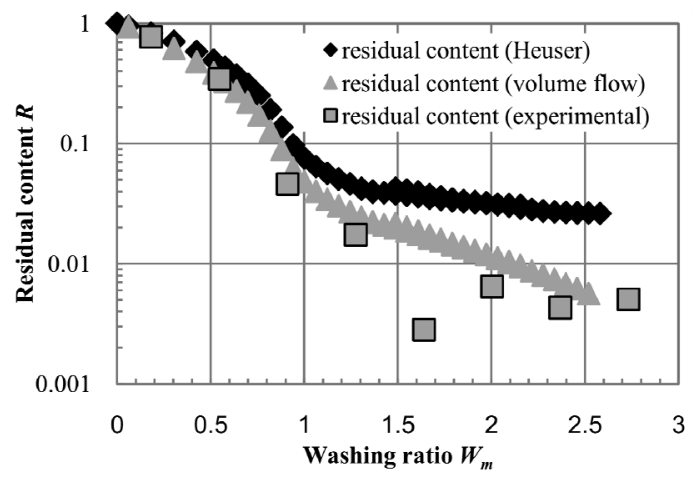

Figure 4. Residual content/concentration ratio in the filter cake for DF600 (hydrophilic); mother liquid methanol, washing liquid water.

\section{Conclusions}

The flushing of filter cakes is an important technique for industrial applications. This works shows the importance of wetting behaviours between the solid system and the mother liquid and washing liquid involved in a flushing process. It has been shown that the washing quality and the washing regimes depend on the surface energy of the system.

Another interesting and not to be undervalued fact is the difference between the detectable concentrations within a filter cake and the calculated concentration (residual content) out of the concentration ratio in the filtrate. An amplification of the residual content by a normalised volume flow eliminates the shift between experiment and calculation to a minimum.

\section{References}

[1] J. Heuser, "Filterkuchenwaschprozesse unter Besonderer Berücksichtigung Physikalisch-Chemischer Einflüsse," Shaker-zgl. Diss. U. Karlsruhe, Aachen, 2003.

[2] W. Bender, "Filtrieren, Auswaschen und Entfeuchten feindisperser Feststoffe," Chemie Ingenieur Technik, Vol. 
48, No. 4, 1976, pp. 270-281. doi:10.1002/cite.330480404

[3] F. Hardekopf, "Experimentelle und Theoretische Untersuchungen zum Waschen von Filterkuchen auf Filterpressen," Chemie Ingenieur Technik, Vol. 64, No. 11, 1992, pp. 1041-1044. doi:10.1002/cite.330641123

[4] F. Russlim, "Flow and Mass Transfer Phenomena in Filter Cake Washing Driven by Mass Force," Dissertation University, Karlsruhe, 2009.

[5] S. Neubauer and U. Peuker, "Flüssigkeitsmischungen als Suspensionsflüssigkeit-Wirkung auf die Filtrationspara-
nmeter,"Chemie Ingenieur Technik, Vol. 79, No. 2, 2007, pp. 1753-1758. doi:10.1002/cite.200700128

[6] M. Wilkens and U. Peuker, "Flushing-Washing Behaviour of Filter Cakes," $13^{\text {th }}$ Nordic Filtration Symposium, Iappeenranta, 10-11 June 2010.

[7] M. Wilkens and U. Peuker, "Filter Cake Washing with Non-Aqueous Liquids - The Influence of the Surface Properties," Filtech Conference 2011, Wiesbaden, 22-24 March 2011. 